EUROPEAN ORGANISATION FOR NUCLEAR RESEARCH

CERN-PPE/95-081

$10^{t h}$ May 1995

\title{
Single Photon and Radiative Events at LEP
}

\author{
Peter Mättig \\ Universität Bonn, 53115 Bonn, Germany
}

\begin{abstract}
Experimental studies of exotic photon production at the LEP $e^{+} e^{-}$collider are summarised. Emphasis is given to the potential signals of anomalous boson self interactions: searches for $Z Z \gamma$ couplings in $\nu \bar{\nu} \gamma$ final states, $Z^{0}$ resonance decays into three photons and the decay $Z^{0} \rightarrow \gamma+$ Higgs. A brief account of studies on possible non-standard fermion-photon couplings is also given.
\end{abstract}

Invited Talk at the Symposium on Vector Boson Self Interactions Los Angeles, California, Feb. 1-3, 1995 


\section{Introduction}

Photon production at LEP allows a broad range of physics, both within and beyond the Standard Model, to be explored. Within the Standard Model photons do not couple directly to the $Z^{0}$. Instead, as depicted in Fig.1, photons originate mainly from incoming (1a) or outgoing (1b) fermions. Particularly in hadronic $Z^{0}$ decays photons may originate from hadron decays like $\pi^{0} \rightarrow \gamma \gamma(1 \mathrm{c})$. In addition, two photons may be produced by an electron t-channel exchange (1d). With the exception of hadron decays these contributions are theoretically understood to high precision and have been used to study QED at the highest energies, electroweak couplings of all fermions and QCD.

The main characteristics of these photons are their typical bremsstrahlung spectra with a preference for small angles $\sim 1 / \alpha_{f, \gamma}$ with respect to the emitting fermion and for low energies $\sim 1 / E_{\gamma}$. On the $Z^{0}$, initial state photons are suppressed due to the large drop in cross section below the resonance. More important for most experimental studies are photons emitted from the outgoing fermions. Photons from hadron decays like $\pi^{0} \rightarrow \gamma \gamma$ in $Z^{0} \rightarrow$ hadrons or $\tau^{+} \tau^{-}$are also emitted dominantly along the fermion direction. They are in general surrounded by other hadrons and can be suppressed by isolation requirements on the photon candidate. Somewhat different is the $e^{+} e^{-} \rightarrow \gamma \gamma$ process, which, in the absence of higher order photon emission (e.g. $1 d)$, leads to monoenergetic photons of beam energy.

Any deviation from these rather strict expectations, either of the photon yield or of their properties, is an unambiguous sign for physics outside the Standard Model. This, together with their rather clear experimental signature, makes photons at LEP a sensitive probe for non-standard physics.

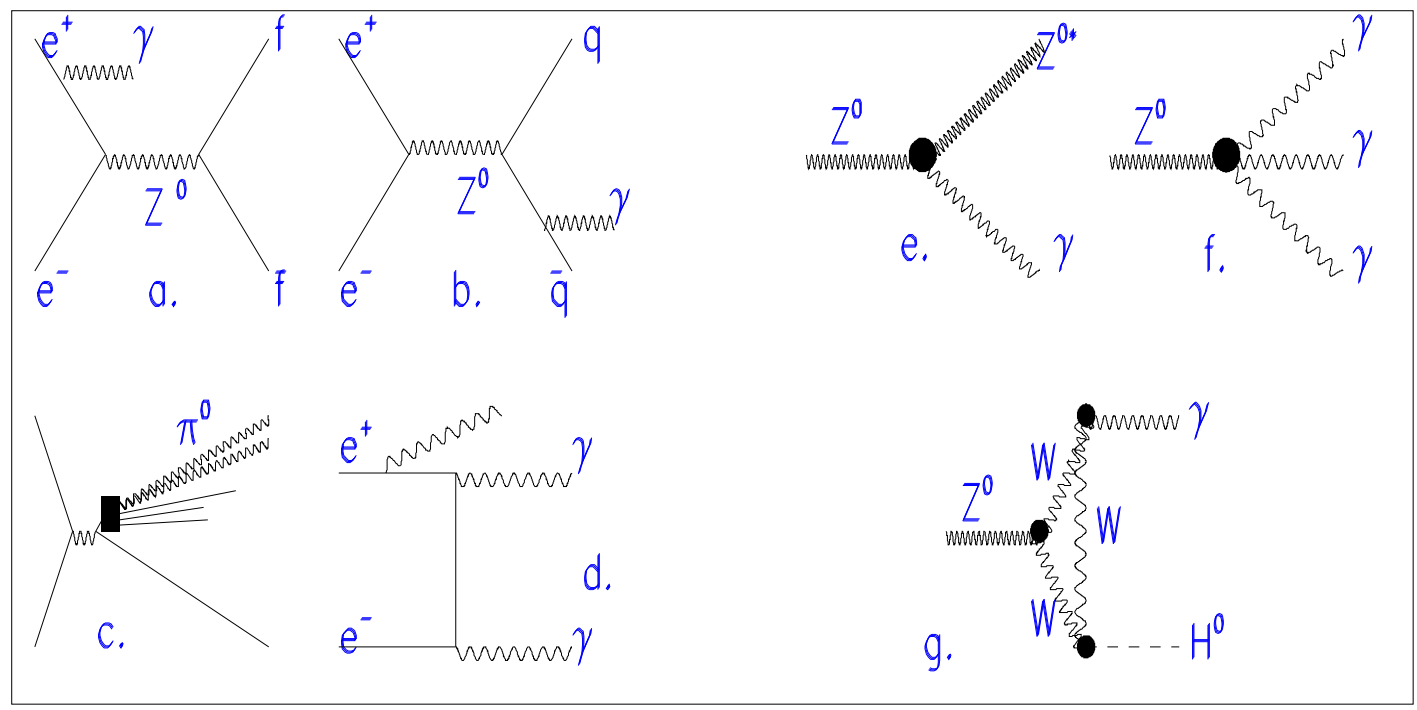

Figure 1: Standard Model photon sources (a-d) and possible nonstandard contributions (f-g).

Radiative events appear in many scenarios of Standard Model extensions, some of which are addressed in [1]. In the context of vector boson self interactions three processes (Fig. $1 \mathrm{f}-\mathrm{g}$ ) are of particular interest. 


\begin{tabular}{|c|c|c|c|c|}
\hline & $\nu \nu \gamma$ & $\mu \mu \gamma$ & $\tau \tau \gamma$ & $q \bar{q} \gamma$ \\
\hline $\begin{array}{l}E_{\gamma}^{\min }(\mathrm{GeV}) \\
\left|\cos \theta_{\gamma}\right|^{\max } \\
\alpha_{f, \gamma}^{\min }(\text { deg. })\end{array}$ & $\begin{array}{l}1.5 \\
0.7\end{array}$ & $\begin{array}{c}2 \\
0.98 \\
5 \\
\end{array}$ & $\begin{array}{c}3 \\
0.94 \\
10 \\
\end{array}$ & $\begin{array}{c}5 \\
0.94 \\
10-15 \\
\end{array}$ \\
\hline $\begin{array}{l}\text { ALEPH } \\
\text { DELPHI } \\
\text { L3 } \\
\text { OPAL }\end{array}$ & $\begin{array}{l}16 \mathrm{pb}^{-1} \\
80 \mathrm{pb}^{-1} \\
40 \mathrm{pb}^{-1}\end{array}$ & $\begin{array}{c}40 \mathrm{pb}^{-1} \\
17 \mathrm{pb}^{-1} \\
7 \mathrm{pb}^{-1}\end{array}$ & $\begin{array}{c}40 \mathrm{pb}^{-1} \\
17 \mathrm{pb}^{-1} \\
7 \mathrm{pb}^{-1}\end{array}$ & $\begin{array}{l}41 \mathrm{pb}^{-1} \\
80 \mathrm{pb}^{-1} \\
40 \mathrm{pb}^{-1} \\
80 \mathrm{pb}^{-1}\end{array}$ \\
\hline Physics & $\begin{array}{c}\nu \text { counting } \\
\nu^{*}, \text { magn.moment }\end{array}$ & $\overline{Z^{\prime}}$ & $\begin{array}{c}Z^{\prime} \\
\text { el., magn. moments }\end{array}$ & $\begin{array}{c}\text { QCD } \\
\text { el.weak }\end{array}$ \\
\hline
\end{tabular}

Table 1: Data samples used at LEP for $f \bar{f} \gamma$ analyses. Typical values are given for cuts on the minimum photon energy $E_{\gamma}^{\text {min }}$, the minimum angle $\theta_{\gamma}$ with respect to the beam direction, and the minimum angle $\alpha_{f, \gamma}^{\min }$ between the outgoing fermion and the photon. The data samples used by the various experiments for published or preliminary analyses are also given.

- Similar to possible $(\gamma Z) W W$ couplings, which will be explored at LEP2, also $(\gamma Z) Z \gamma$ couplings may be anomalous. On the $Z^{0}$ especially the decay $Z^{0} \rightarrow Z^{0 *} \gamma$ is potentially rewarding (Fig.1e) with a sensitivity to anomalous $Z Z \gamma$ couplings.

- The Standard Model also inhibits a $Z^{0}$ decay into three photons at the tree level. Such a decay may occur if the $Z^{0}$ is composite (Fig.1f).

- Within the Standard Model photons may be produced e.g. together with a Higgs boson by the loop correction displayed in Fig.1g. For Higgs masses smaller than $m_{Z^{0}}$ and anomalous boson couplings this contribution may become observable at LEP.

After summarising general aspects of the LEP photon samples, we will discuss the experimental studies of these processes at LEP in more detail.

\section{The LEP Data}

As of the end of '94 each of the LEP experiments has collected data samples of about 140 $\mathrm{pb}^{-1}$ or 5.5 million $Z^{0}$ decays. In general only a small fraction of these has been publicized in searches for beyond the Standard Model effects. For the $f \bar{f} \gamma$ analyses the statistics used by the various experiments, typical cuts, and some principle physics motivations are listed in in Table.

In general LEP experiments identify photons in most of the phase space. Some restrictions apply to the $\nu \nu \gamma$ and $q \bar{q} \gamma$ channels. For the neutrino analysis events with just a single photon 


\begin{tabular}{||l|c|c|c|c||}
\hline \hline Experiment & Ref. & $\mathcal{L}_{e f f}$ & $E_{\gamma}>15 \mathrm{GeV}$ & $E_{\gamma}>23 \mathrm{GeV}$ \\
ALEPH & {$[5]$} & $6.3 \mathrm{pb}^{-1}$ & $0($ exp. 0) & 0 (exp. 0) \\
L3 & {$[4]$} & $35.0 \mathrm{pb}^{-1}$ & $9(\exp .8)$ & 1 (exp. 1.2) \\
OPAL & {$[6]$} & $16.7 \mathrm{pb}^{-1}$ & $5(\exp .1 .4)$ & $0($ exp. 0) \\
\hline Combined & & $48.0 \mathrm{pb}^{-1}$ & $14(\exp .9 .4)$ & 1 (exp. 1.2) \\
\hline \hline
\end{tabular}

Table 2: Number of high energy single photons observed (and expected).

in the detector are selected. Since up to now the main goal has been the counting of neutrino species, the experimental emphasis was on low energy photons. To make sure that no particle evades detection in the blind area of the beam pipe, photons at a large polar angle are selected. In the case of $q \bar{q} \gamma$ the main experimental challenge is to suppress the $\pi^{0}$ background. This is achieved by requiring the photon candidate to be fairly energetic and isolated. This implies a large $\alpha_{f, \gamma}$.

\section{Searching for Anomalous $Z Z \gamma$ Couplings}

In its most general form the $Z^{0} \rightarrow Z^{0 *} \gamma$ production can be expressed [2] by four form factors $h_{i}$. All of them violate charge conjucation, two of them $(i=1,2)$ are $\mathrm{CP}$ violating, the other two are CP conserving. Neglecting the charge of the fermions, the cross sections are identical for $h_{1}=h_{3}\left(\right.$ and $\left.h_{2}=h_{4}\right)$. In the Standard Model $h_{i}=0$. In its extensions at some typical scale $\Lambda$, the $h_{i}$ are expected to be $\mathcal{O}\left(m_{Z^{0}}^{4} / \Lambda^{4}\right)$ for $s<<\Lambda^{2}$.

At LEP the potential signature for an anomalous coupling is an excess of photons in the process $e^{+} e^{-}\left(\rightarrow Z^{0}\right) \rightarrow \gamma f \bar{f}$ where the $f \bar{f}$ are fermion pairs produced with a composition of the standard $Z^{0}$ decays (i.e. $\sim 20 \% \nu \bar{\nu}, \sim 10 \% l^{+} l^{-}, \sim 70 \% q \bar{q}$ ). Within the cuts of $\alpha_{f, \gamma}>100$ mrad, $\left|\cos \theta_{\gamma}\right|<0.95$, and $E_{\gamma}>5 \mathrm{GeV}$, typically used at LEP, anomalous couplings would, according to the calculation of [3], lead to cross sections (given in $\mathrm{pb}$ )

$$
\sigma_{\text {anomalous }} \sim 1.7\left|h_{3}\right|^{2} B R\left(Z^{0} \rightarrow f \bar{f}\right) \quad \text { or } \quad \sim 0.25\left|h_{4}\right|^{2} B R\left(Z^{0} \rightarrow f \bar{f}\right)
$$

which has to be compared to the Standard Model background of

$$
\sigma_{I S R} \sim 45 \cdot B R\left(Z^{0} \rightarrow f \bar{f}\right) \quad \text { and } \quad \sigma_{F S R} \sim 2400 \cdot e_{f}^{2} \cdot B R\left(Z^{0} \rightarrow f \bar{f}\right)
$$

here $e_{f}$ is the electric charge of the outgoing fermion.

The polar angle distributions of initial and final state radiation and for an anomalous coupling $\left|h_{3}\right|=5$ are shown in Fig.2a. A cut on the polar angle like $|\cos \theta|<0.8$ suppresses the contribution from ISR significantly. The resulting distribution of the photon energy is shown in Fig.2b. Applying an additional cut on the photon energy like $2 E_{\gamma} / E_{c m}>0.5$, i.e. $E_{\gamma}>23 \mathrm{GeV}$, 

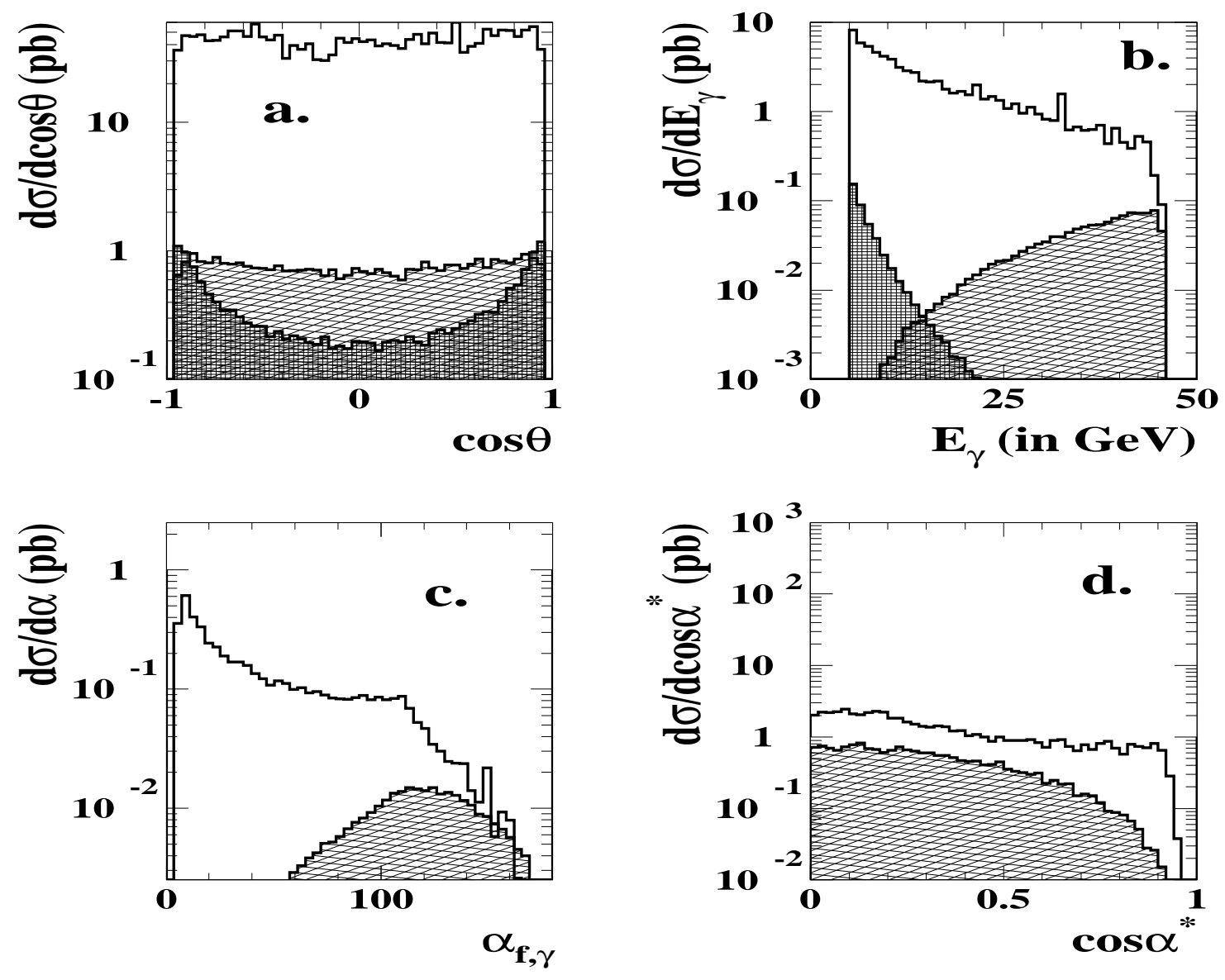

Figure 2: Properties of various photon sources in the $\mu \mu \gamma$ final state. The open histogram shows the FSR and the dark histogram the ISR contributions. An anomalous coupling of $\left|h_{3}\right|=5$ would lead to the cross hatched distribution. Distributions are shown after incremental cuts: a. polar angle $\cos \theta$ with wide cuts (see text); b. photon energy $E_{\gamma}$ after $\left|\cos \theta_{\gamma}\right|<0.8$; c. minimum angle $\alpha_{f, \gamma}$ for $E_{\gamma}>23 \mathrm{GeV}$; d. angle $\alpha^{*}$ in fermionic rest system after cut on $\alpha_{f, \gamma}>100$ degrees.

eliminates the ISR contribution almost completely, while hardly affecting anomalous contributions. As shown in Fig.2c and $d$ the much more abundant FSR background is much harder to suppress and only at the price of a substantial loss in efficiency for anomalous production. Since it is free of final state photons, the $\nu \bar{\nu} \gamma$ channel becomes the outstanding mode for testing for anomalous $Z Z \gamma$ couplings.

The photon energy spectrum of L3 [4] is shown in Fig.3a together with the Standard Model expectation and an expectation for an anomalous coupling $\left|h_{3}\right|=1$ which leads to high energy photons. Together with the results from ALEPH [5], and OPAL [6] the observed yields for $E_{\gamma}>15$ and $23 \mathrm{GeV}$ are listed in Table 2. Also listed is the effective luminosity

$$
\mathcal{L}_{e f f}=\epsilon_{\gamma} \sum_{i} \frac{\sigma\left(E_{c m}^{i}\right)}{\sigma\left(M_{Z^{0}}\right)} \mathcal{L}_{n o m}^{i}
$$

where $\mathcal{L}_{\text {nom }}^{i}$ is the experimental luminosity collected at the c.m. energy $E_{\mathrm{cm}}^{i}$. These luminosities are weighted by the ratio of the cross sections at this energy and at the $Z^{0}$ peak, $\epsilon_{\gamma}$ is the 


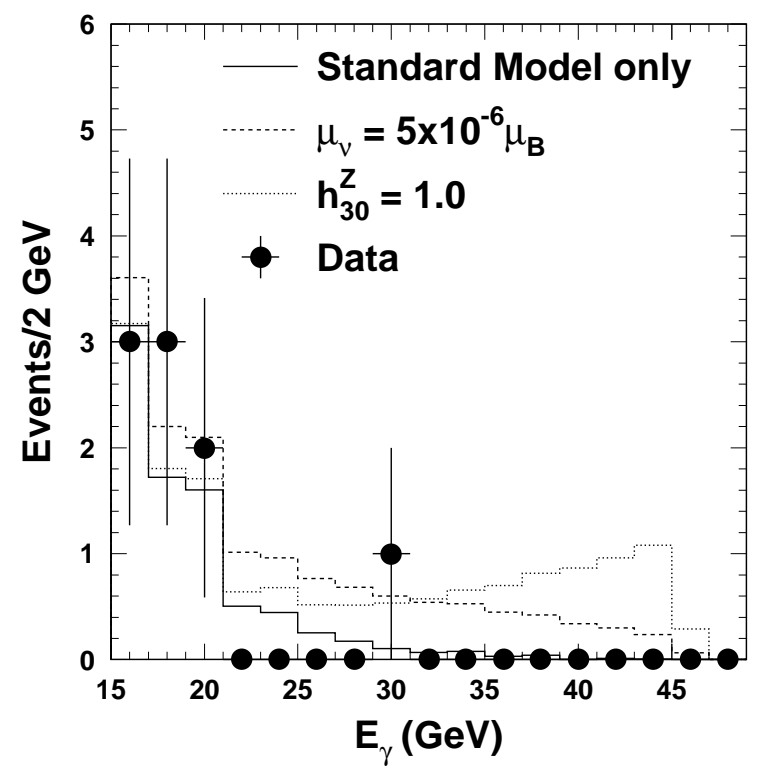

(a)

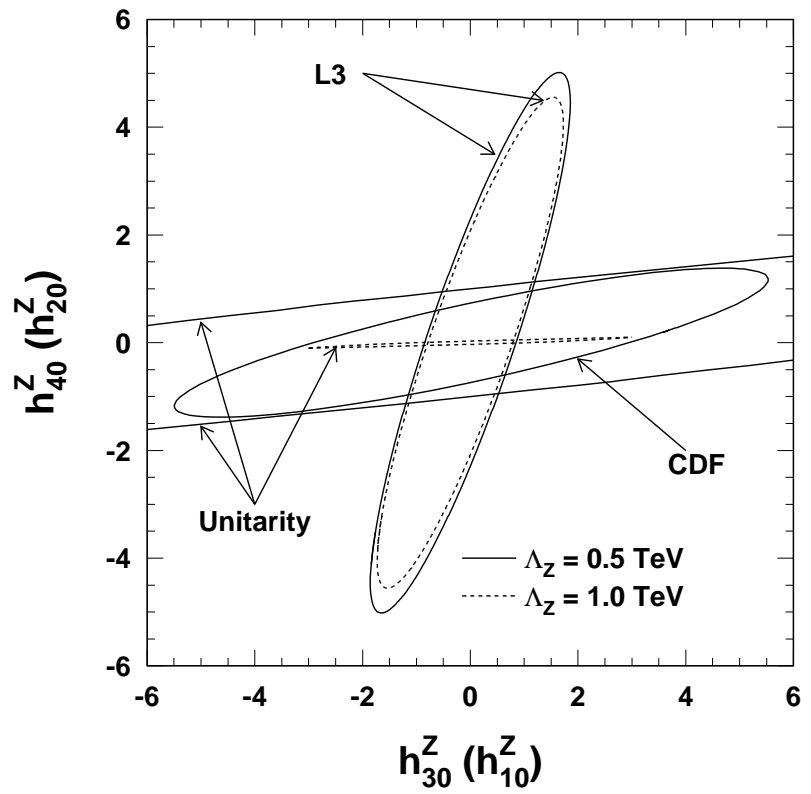

(b)

Figure 3: (a) Photon energy spectrum for $\nu \bar{\nu} \gamma$ events. (b) Allowed region of anomalous couplings $h_{3}\left(h_{1}\right)$ and $h_{2}\left(h_{4}\right)$ from both LEP and the Tevatron. Both figures from ref [4].

experimental photon detection efficiency.

No significant excess of high energy photons is observed. Based on the LEP data sample the production of more than four $\gamma Z^{0 *}$ events with an energy of more than $23 \mathrm{GeV}$ can be excluded at $95 \%$ C.L.. This can be interpreted as

$$
\left|h_{1,3}^{Z}\right|<0.73 \quad\left|h_{2,4}^{Z}\right|<1.96
$$

Here it was assumed that just one coupling constant contributes. In principle the two either $\mathrm{CP}$ conserving or violating contributions could interfer. The allowed region in the $\left(h_{3}, h_{4}\right)$ and $\left(h_{1}, h_{2}\right)$ parameter space as obtained by L3 is displayed in Fig. 3b. Also shown are the corresponding limits from $Z \gamma$ final states as observed by CDF [7]. In comparing LEP and Tevatron limits a few differences have to be noted. Firstly, the $p \bar{p}$ result does not discriminate between the $\gamma \gamma Z$ and $Z Z \gamma$ couplings. Secondly, at LEP the couplings are determined at a fixed c.m. energy where the sensitivity to $h_{2}\left(h_{4}\right)$ is less than the one to $h_{1}\left(h_{3}\right)$. At the Tevatron the limits are derived from the energy dependence of the production. Since the anomalous contributions grow like $\left(E_{c m} / M_{Z}\right)^{3}$ and $\left(E_{c m} / M_{Z}\right)^{5}$ for the two coefficients, one is more sensitive to $h_{2}\left(h_{4}\right)$. As a result the LEP and Tevatron limits are rather orthogonal. This energy dependence at the Tevatron also requires them to introduce a form-factor behaviour to avoid unitarity violation. CDF uses $h_{i}=h_{i}^{0} /\left(1+s / \Lambda^{2}\right)^{n}$, with $n=3$ for $i=1,3$, and $n=4$ for $\mathrm{i}=2,4$ for some assumed values of $\Lambda$.

Combining LEP and CDF, limits of $\mathcal{O}(1)$ are obtained for $\left|h_{i}\right|$. This is the first direct constraint on a potential anomalous $(Z, \gamma) Z \gamma$ coupling, which is, however, still several orders of magnitude above the expectation for some new effects. 
Based on these numbers one may estimate the final potential of LEP1 ${ }^{1}$. At the end of '95, running at the $Z^{0}$ pole will be completed and some $200 \mathrm{pb}^{-1}$ will be collected per experiment. Allowing for an improved efficiency for high energy photons of $\sim 70 \%$ and taking into account the distribution of c.m. energies around the $Z^{0}$-pole, we expect a $\sim$ tenfold increase in effective luminosity from the combination of all experiments. The sensitivity to anomalous couplings will then improve by $\sim \sqrt{10}$ to $\left|h_{1,3}\right|<0.2$ and $\left|h_{2,4}\right|<0.6$.

\section{The Fermion - Photon Coupling}

Apart from searching for anomalous boson self couplings, the photon spectra may also be interpreted in terms of fermion - photon couplings. For example, the $\nu \bar{\nu} \gamma$ measurement [4] has been used by L3 to set limits on an anomalous magnetic moment of the $\tau$ neutrino of $\mu_{\nu_{\tau}}<4.1 \cdot 10^{-6} \mu_{B}$ with $\mu_{B}$ the Bohr magneton. Similar analyses can be performed for charged leptons.



(c)



Calculation/Data

Figure 4: (a) Photon energy spectrum in $\tau \tau \gamma$ events [8]. (b) Ratio theory/data for isolated photon production in hadronic events. The light grey region indicates the one standard deviation range of the LEP average with only the experimental uncertainties. For the dark grey area theoretical uncertainties are included.

The photon energy spectrum for $\tau$ pairs from a preliminary measurement of DELPHI [8] is

\footnotetext{
${ }^{1}$ The prospects at LEP2 are addressed by Busenitz at this conference.
} 
shown in Fig.4a to be in agreement with the Standard Model expectation. Using the calculation of [9], anomalous magnetic or electric moments $F_{2}^{\tau}$ and $F_{E D M}^{\tau}$ of $\tau$ 's would lead to an essentially energy independent distribution. The observed distribution can be translated into

$$
F_{2}^{\tau}\left(q^{2}=0\right)<0.072 \quad F_{E D M}^{\tau}\left(q^{2}=0\right)<4 \cdot 10^{-16}
$$

both at $95 \%$ confidence. These measurements complement the tighter bounds obtained from $\tau$ pair production at $q^{2}>\left(2 m_{\tau}\right)^{2}$ in the $e^{+} e^{-}$continuum.

The $\mu \mu \gamma$ events were used by OPAL [10] and DELPHI [11] to study event properties at c.m. energies several $\mathrm{GeV}$ below the pole. No deviation from the Standard Model expectations are observed allowing limits to be set on possible additional $Z^{\prime}$ gauge bosons.

Because of its wide potential for QCD and electroweak quark studies [12] photon bremsstrahlung from quarks has been rather actively scrutinized at LEP (for a review see [13]). All experiments have compared isolated photon production with matrix element calculations of $\mathcal{O}\left(\alpha \alpha_{s}\right)$. In Fig. 4 b the ratio $R_{q \bar{q} \gamma}$ of the calculation [14] over the data [15] are displayed. The selection criteria for the various experiments are slightly different but lead to consistent values of $R_{q \bar{q} \gamma}$ which can be averaged to yield

$$
R_{q \bar{q} \gamma}=\frac{\sigma_{\text {theory }}}{\sigma_{\text {measurement }}}=1.077 \pm 0.042 \pm 0.04
$$

indicating agreement with the Standard Model. The two uncertainties are due to the experimental analysis and to the theoretical calculation, particularly the value of $\alpha_{s}$, the dependence of the cut - off against the quark - photon singularity and hadronisation [16].

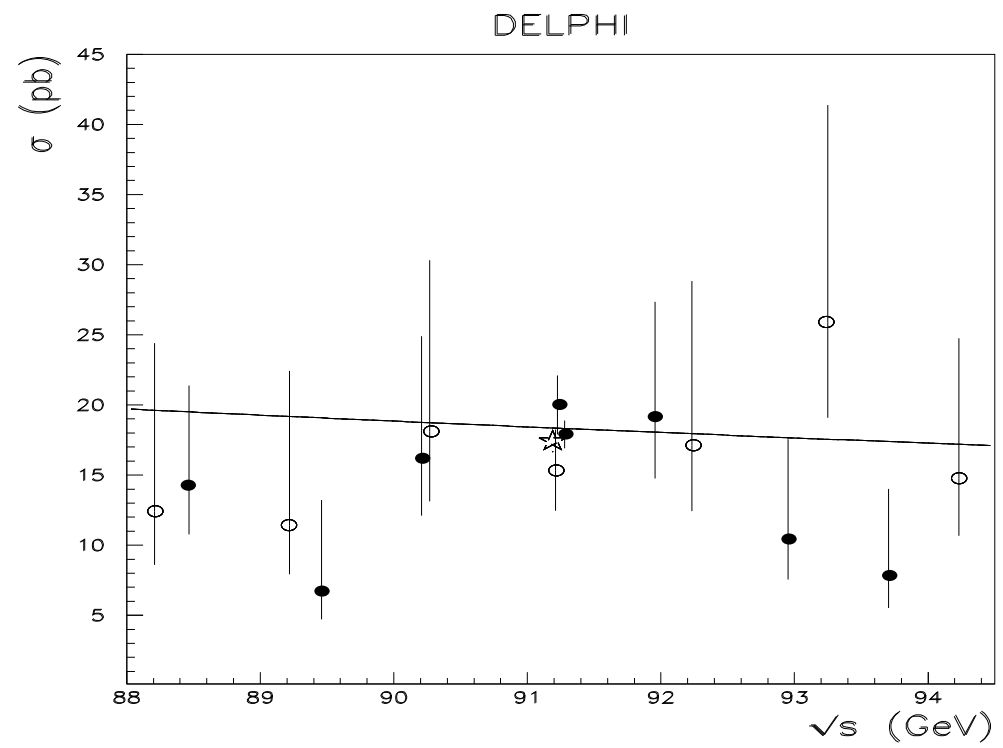

Figure 5: Cross section for $e^{+} e^{-} \rightarrow \gamma \gamma(\gamma)$ in the central region of the DELPHI detector [18]. Open and full circles represent data collected in different years, the line is the QED expectation. 


\section{Searching for $Z^{0} \rightarrow \gamma \gamma \gamma$}

Whereas the $Z^{0}$ decay into two photons is forbidden due to Yang's theorem, it is the Standard Model dynamics that prohibits the decay of the $Z^{0}$ into three photons at tree level. Loop contributions give rise to $\mathrm{BR}\left(Z^{0} \rightarrow \gamma \gamma \gamma\right) \sim \mathcal{O}\left(10^{-10}\right)$, much below the sensitivity of current LEP experiments. $Z^{0}$ decays into three photons may be largely enhanced in extensions of the Standard Model, if, for example, the $Z^{0}$ is built up from charged constituents of size $1 / \Lambda$. Its charge may be 'felt' by the photon depending on its resolution power $1 / E_{c m}$. According to [17], within such models

$$
\Gamma\left(Z^{0} \rightarrow \gamma \gamma \gamma\right)=\frac{64 \alpha^{3}}{9}\left(\pi^{2}-9\right) M_{Z} \frac{|\phi(0)|^{2} N_{c} N_{H}<Q^{6}>}{4 \pi M_{Z}^{3}}
$$

where $|\phi(0)|$ is the value of the wave function at the origin, $N_{c}$ and $N_{H}$ are the number of colours and hypercolours of the constituents and $Q$ their electric charge. This leads to estimates of $\operatorname{BR}\left(Z^{0} \rightarrow \gamma \gamma \gamma\right) \sim 2 \cdot 10^{-4}<Q^{6}>$

Standard Model background to this resonance decay is the pure QED process (Fig.1d) via a t-channel electron exchange with an additional photon emitted from the incoming electron. This cross section decreases like $\sim 1 / E_{c m}^{2}$. A direct $Z^{0}$ decay, however, should show up as a resonance structure of the cross section. No such enhancement is observed, see, for example, Fig.5 of ref. [18].

Alternatively the direct $Z^{0} \rightarrow \gamma \gamma \gamma$ decay could be established from the energy spectrum of the least energetic photon. For the Standard Model background its energy should be distributed according to the $1 / E_{\gamma}$ bremsstrahlung spectrum. Assuming for the direct decay a phase space distribution, the energy of the least energetic photon tends towards $M_{Z} / 3$. Such a search has been performed by L3 [19]. The most restrictive limits on direct $Z^{0}$ decays are, at $95 \%$ confidence,

$$
B R\left(Z^{0} \rightarrow \gamma \gamma \gamma\right) \quad<1.7 \cdot 10^{-5}(\mathrm{DELPHI}) ; \quad<1 \cdot 10^{-5}(\mathrm{~L} 3)
$$

\section{Anomalous Higgs Production}

Searches for the Standard Model Higgs boson at LEP have up to now been based on the Bjorken process $Z^{0} \rightarrow Z^{0 *} H^{0}[20]$ allowing limits of $m_{H}>64.5 \mathrm{GeV}$ to be set [21]. An alternative Higgs production mechanism (Fig. $1 \mathrm{~g}$ ) is the decay $Z^{0} \rightarrow \gamma H^{0}$ which proceeds dominantly via $W$ loops [22]. Because of the larger phase space its $Z^{0}$ branching ratio exceeds the one of the Bjorken mechanism for $m_{H}>70 \mathrm{GeV}$, albeit its branching ratio of $10^{-6}-10^{-7}$ is too small to be observed at LEP.

It may increase if the couplings among bosons are anomalous. Expressing the new interactions at some scale $\Lambda$ by an effective Lagrangian, one finds [23]

$$
\Gamma\left(Z^{0} \rightarrow H^{0} \gamma\right)=\frac{\alpha}{96 m_{Z}}\left(m_{Z}^{2}-m_{H}^{2}\right)^{3}\left|c_{S M}+\frac{\delta_{n e w}}{\Lambda^{2}}\right|^{2}
$$




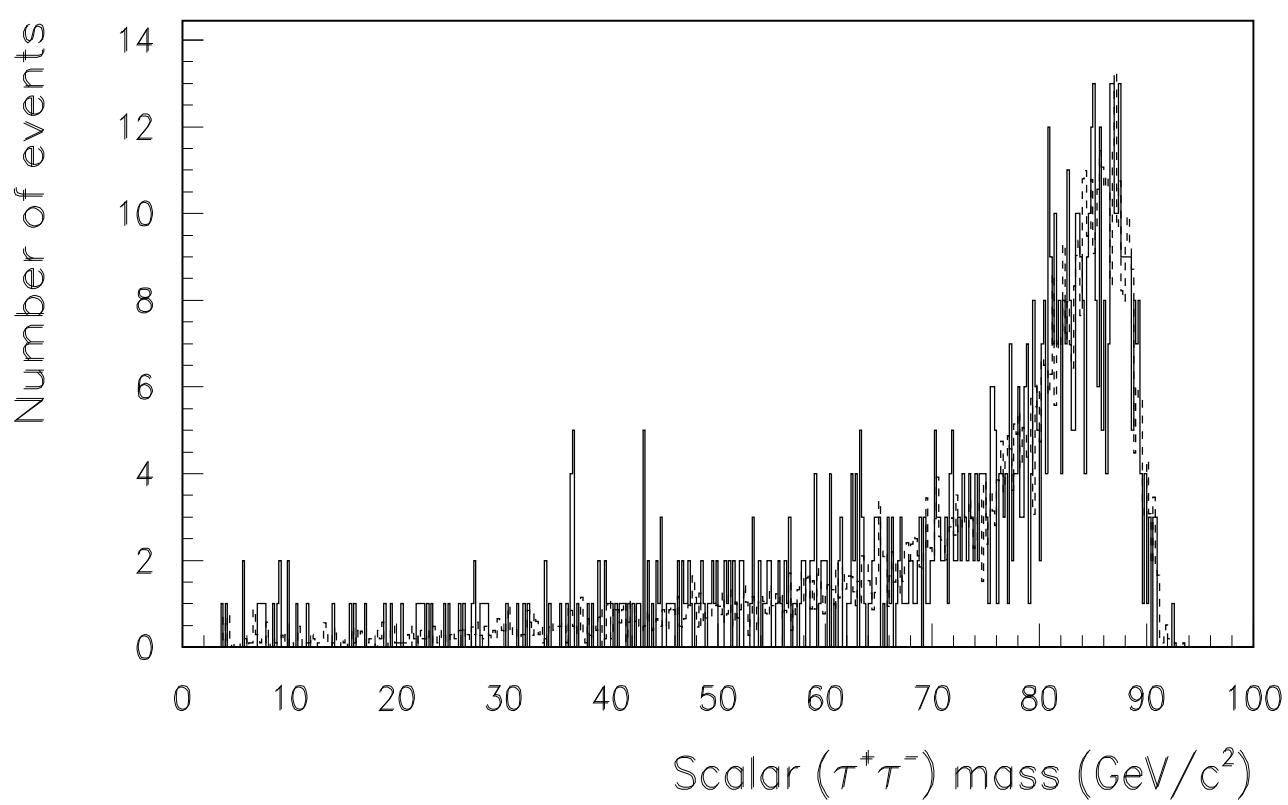

Figure 6: Invariant mass of the $\tau^{+} \tau^{-}$system produced in association with a photon $[26]$.

where $c_{S M}$ denotes the Standard Model contribution and $\delta_{\text {new }} / \Lambda^{2}$ parametrises the contribution from new boson interactions. Even taking into account constraints from measurements at low energy, from LEP and from the Tevatron, these non Standard Model contributions may increase the $H^{0} \gamma$ production by several orders of magnitude while at the same time weaken the standard Higgs limits.

The decay rates of a Standard Model Higgs boson are unambigously predicted. For the range of $m_{H}$ of $12-80 \mathrm{GeV}$, relevant for the current Higgs searches at LEP, $85 \%$ of the Higgses should decay into a beauty pair. Extensions of the Standard Model may imply different branching ratios. For example, anomalous boson couplings could enhance the decay branching ratio $H^{0} \rightarrow$ $\gamma \gamma$ to even become the dominant decay mode. At LEP this would lead to a resonant $\gamma \gamma$ state with associated production of fermion pairs. A couple of years ago, the L3 collaboration claimed an excess of this kind in $\left(\mu^{+} \mu^{-}, e^{+} e^{-}\right) \gamma \gamma$ events with $M_{\gamma \gamma} \sim 60 \mathrm{GeV}$ [24]. With increased statistics and extending the search to other channels this observation was not confirmed by the other LEP experiments [25].

All LEP experiments have performed searches for a narrow resonance in the channel $\gamma f \bar{f}$ with $f$ being either neutrinos, charged leptons or quarks. The mass of the fermionic system recoiling against the photon can either be reconstructed from the photon energy alone using

$$
M_{\text {recoil }}=E_{c m} \sqrt{1-2 E_{\gamma} / E_{c m}}
$$

or from kinematical fits exploiting the precise knowledge in $e^{+} e^{-}$collisions of the total c.m. energy and momentum $\left(\sum \vec{p}=0\right)$. These methods allow a good mass resolution even if the scalar particle decays hadronically yielding $\delta M_{\text {recoil }} \sim 1 \mathrm{GeV}$ for large masses and $\sim 2-3 \mathrm{GeV}$ for small masses.

To suppress the principle background from final state bremsstrahlung photons, angular cuts can be imposed. For example, one can use $\alpha^{*}$, the angle between the fermion and the photon 


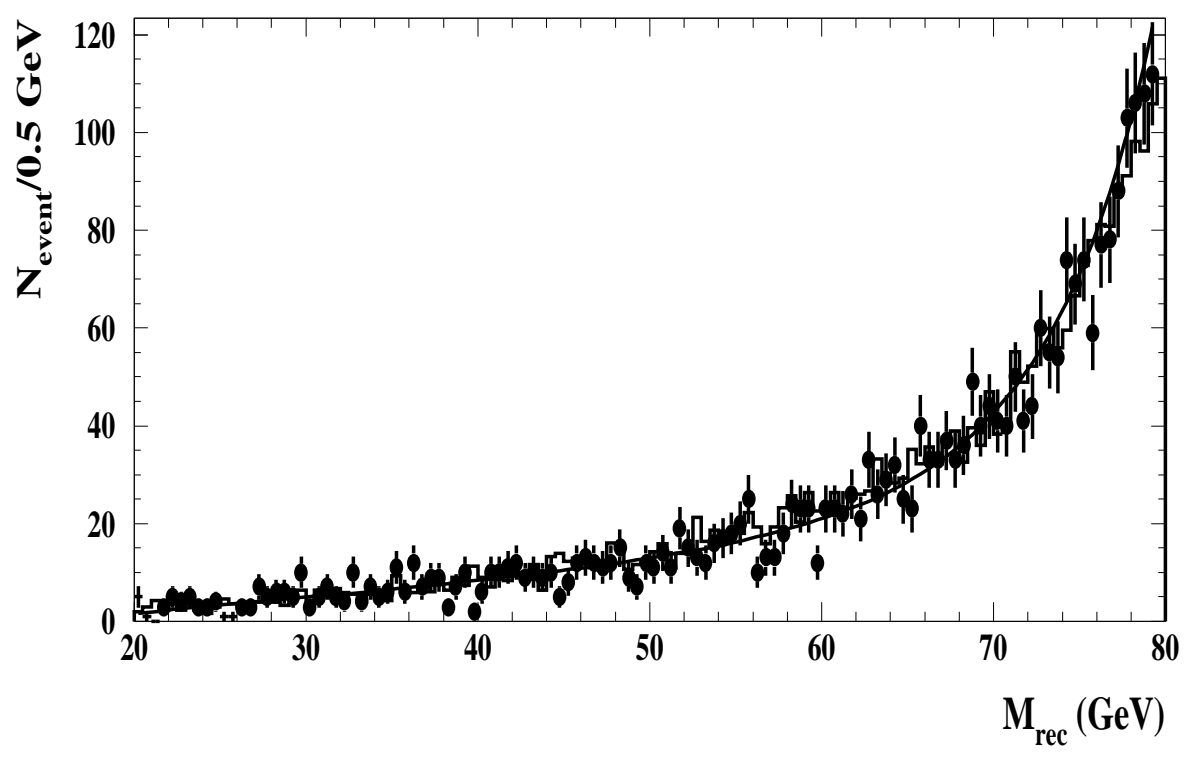

Figure 7: Invariant mass of the hadronic system produced in association with a photon [27].

in the fermionic rest system. Whereas bremsstrahlung photons tend to be aligned with the emitting fermion, a Higgs decay leads to an isotropic distribution.

Preliminary spectra of the $\tau$ pair [26] and hadronic recoil masses [27], based on 1.1, respectively 1.8 million hadronic $Z^{0}$ decays, are shown in Figs.6,7. No resonance structure is observed in either of these distributions. The spectra can be interpreted in terms of limits on the product branching ratios $\mathrm{BR}\left(Z^{0} \rightarrow S^{0} \gamma\right) \times \mathrm{BR}\left(S^{0} \rightarrow f \bar{f}\right)$ at $95 \%$ confidence level as being smaller than typically $\sim 5 \cdot 10^{-6}$ for charged leptons [26] (Fig.8) and $\sim 3 \cdot 10^{-5}$ for quarks [27, 28].

Given the probable preference of the scalar to decay into beauty quarks, one can improve limits by invoking beauty tagging. Selecting hadronic events with a secondary vertex, beauty events can be enriched with a rather high efficiency. Tagging of $b \bar{b} \gamma$ events has been used by OPAL [27] and DELPHI [28] who retain 60 (20) \% of all beauty events while rejecting 90 (95) $\%$ of events with final state photon radiation from other quark species. The spectrum from OPAL is shown in Fig.9. No significant resonance structure is observed. The result can be interpreted as (see Fig.10)

$$
B R\left(Z^{0} \rightarrow S^{0} \gamma\right) \times B R\left(S^{0} \rightarrow b \bar{b}\right)<2-3 \cdot 10^{-5}
$$

which restricts some of the allowed parameter region used in the calculation of [23] or for a composite Higgs [29].

Combining the results of the LEP experiments and assuming a 2-4 fold increase in statistics until the end of LEP1, limits of $5 \cdot 10^{-6}$ for $b \bar{b}$ and some factor five less for leptons are in reach. 


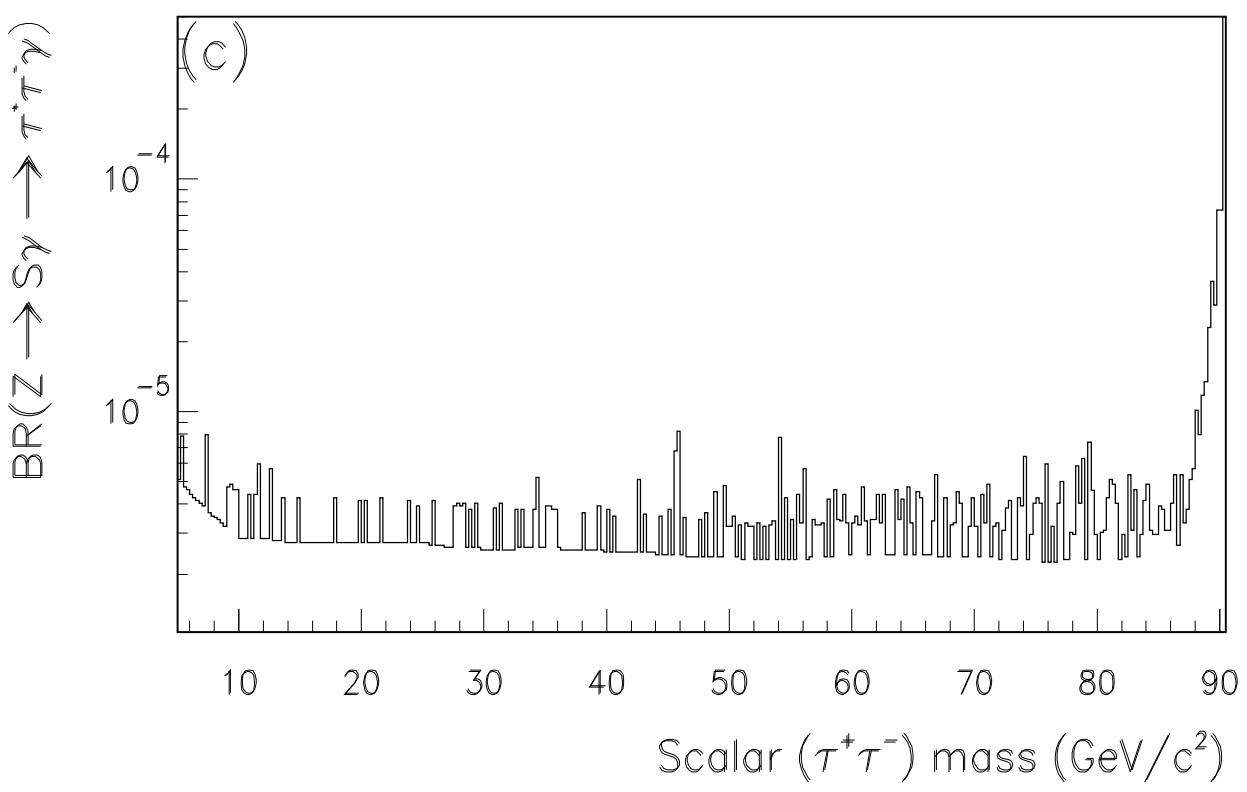

Figure 8: Lower limits at $95 \% \mathrm{CL}$ for the branching ratio $\mathrm{BR}\left(Z^{0} \rightarrow S^{0} \gamma\right)$ $\operatorname{BR}\left(S^{0} \rightarrow \tau^{+} \tau^{-}\right)$as a function of the $\tau^{+} \tau^{-}$mass [26].

\section{Conclusions}

Anomalous photon production is predicted in various extensions of the Standard Model and has been searched for in many different ways at LEP. On the basis of typically $40-50 \mathrm{pb}^{-1}$ no signal has yet been observed.

Using the $Z \rightarrow \nu \bar{\nu}$ decay, limits are derived for anomalous $Z Z \gamma$ couplings of

$$
\left|h_{1,3}^{Z}\right|<0.73 \quad\left|h_{2,4}^{Z}\right|<1.96
$$

Photon production in events with charged leptons and quarks also agree with the Standard Model expectation allowing various new measurements particularly for the $\tau$. The respective limits on an anomalous magnetic moment of the $\nu_{\tau}$, of the $\tau$ itself and the electric dipole moment of the $\tau$ are

$$
\mu_{\nu_{\tau}}<4.1 \cdot 10^{-6} \mu_{B} \quad F_{2}^{\tau}\left(q^{2}=0\right)<0.072 \quad F_{E D M}^{\tau}\left(q^{2}=0\right)<4 \cdot 10^{-16}
$$

Negative searches have been performed on resonant $\gamma \gamma \gamma$ production leading to limits of

$$
\Gamma\left(Z^{0} \rightarrow \gamma \gamma \gamma\right) / \Gamma\left(Z^{0}\right)<1 \cdot 10^{-5}
$$

Finally, studies on scalar resonance production in conjunction with $\gamma$ emission have been made. No narrow signal has been observed and limits on the product branching ratio $\operatorname{BR}\left(Z^{0} \rightarrow\right.$ $\left.S^{0} \gamma\right) \operatorname{BR}\left(S^{0} \rightarrow f \bar{f}\right)$ of $\sim 5 \cdot 10^{-6}$ (charged leptons) and $\sim 2 \cdot 10^{-5}$ (hadrons, beauty pairs) have been set. 




Figure 9: Invariant mass of the $b \bar{b}$ system produced in association with a photon [27].

\section{Acknowledgement}

I wish to thank Ulrich Baur, Steve Errede and Thomas Müller for organising an excellent and inspiring symposium. I am grateful to many of my LEP colleagues for providing me with the most recent results of their experiment and Ulrich Baur for letting me use his program to calculate effects of anomalous $Z Z \gamma$ couplings. In writing up the talk I profited from several comments by Fawzi Boudjema and Graham Wilson.

\section{References}

[1] Workshop on Photon Radiation from Quarks, Annecy 2-3 December 1991, ed. S.Cartwright, CERN 92-04.

[2] K.Hagiwara et al. Nucl.Phys. B282 253 (1987).

[3] U.Baur and E.Berger, Phys.Rev. D47 4889 (1993)

[4] L3-Collaboration, M.Acciarri et al., Phys.Lett. B346 190 (1995).

[5] ALEPH-Collaboration, D.Buskulic et al., Phys.Lett. B313 520 (1993).

[6] OPAL-Collaboration, R.Akers et al., Z.Phys. C65 47 (1995).

[7] CDF-Collaboration, F.Abe et al., FERMILAB Pub-94/244-E and Phys.Rev.Lett. 741941 (1995)

[8] DELPHI-Collaboration, Internal note DELPHI 94-89 PHYS 406. 


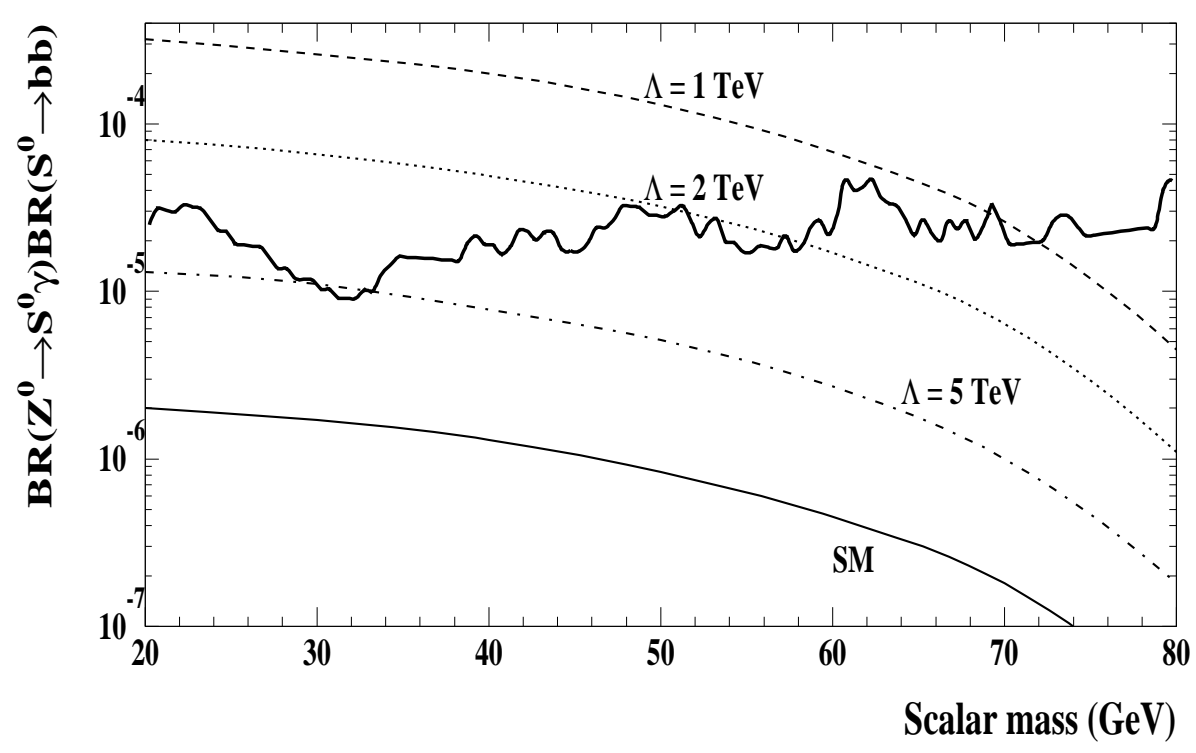

Figure 10: Lower limits at 95\% CL for the branching ratio $\operatorname{BR}\left(Z^{0} \rightarrow\right.$ $\left.S^{0} \gamma\right) \operatorname{BR}\left(S^{0} \rightarrow b \bar{b}\right)$ as a function of the scalar mass (thick full line). Also given is the Standard Model expectation (thin full line) and those for a composite Higgs assuming various values of the composite scale $\Lambda$.

[9] J.A.Grifols and A.Mendez, Phys.Lett. B255 611 (1991).

[10] OPAL-Collaboration, P.D.Acton et al., Phys.Lett. B268 122 (1991).

[11] DELPHI-Collaboration, P.Abreu et al., CERN-PPE 94/121.

[12] P.Mättig and W.Zeuner, Z.Phys. C52 31 (1991).

[13] P.Mättig in Proceedings of the Eighth Lake Louise Winter Institute, Lake Louise, Alberta, Canada, 21-27 February 1993, eds. A. Astbury et al.

[14] Here the calculation of G.Kramer and H.Spiesberger in [1] was used. See also E.W.N.Glover and J.Stirling, Phys.Lett. B295 128 (1992) and Z.Kunszt and Z.Trocsanyi, Nucl.Phys. B394 139 (1993).

[15] ALEPH-Collaboration 93-111 PHYSIC 93-092; DELPHI-Collaboration to be published; L3-Collaboration O.Adriani et al., Phys.Lett. B301 136 (1993); OPAL-Collaboration P.Acton et al., Z.Phys. C58 405 (1993).

[16] P.Mättig, H.Spiesberger and W.Zeuner, Z.Phys. C60 613 (1995).

[17] F.Renard, Phys.Lett. B116 264 (1982).

[18] DELPHI-Collaboration, P.Abreu et al., Phys.Lett. B327 386 (1994).

[19] L3-Collaboration, M.Acciarri et. al., Phys.Lett. B345 609 (1995).

[20] J.D.Bjorken, in Proceedings of the 1976 Summer Institute on Particle Physics, Stanford, ed. M.C.Zipf (SLAC, Stanford CA, 1977) pg.1. 
[21] F.Richard in Proceedings of the XXVII International Conference on the High Energy Physics 20-27 July 1994 Glasgow, Scotland, UK ed. P.J.Bussey and I.G.Knowles.

[22] R.N.Cahn, M.S.Chanowitz and N.Fleishon, Phys.Lett. B82 112 (1979).

[23] K.Hagiwara, R.Szalapski and D.Zeppenfeld, Phys.Lett. B318 155 (1993).

[24] L3-Collaboration, O.Adriani et al., Phys.Lett. B295 337 (1992).

[25] G.W.Wilson in Proceedings of the International Europhysics Conference on High Energy Physics, Marseille, France (July 1993) eds. J.Carr and M.Perrotet.

[26] ALEPH-Collaboration, submitted to the $27^{\text {th }}$ International Conference on High Energy Physics, Glasgow, Scotland, 20-27 July 1994.

[27] OPAL-Collaboration, Internal note OPAL-PN-140.

[28] DELPHI-Collaboration, Internal note DELPHI 94-119 PHYS 436.

[29] D.Düsedau and J.Wudka, Phys.Lett. B180 290 (1986) 